\title{
La marge de manœuvre interprétative chez le cinéaste David Lynch : de Lost Highway à Inland Empire
}

Jean-Michel Denizart

\section{CpenEdition}

Journals

Édition électronique

URL : http://journals.openedition.org/lcc/653

DOI : $10.4000 /$ lcc. 653

ISSN : 2430-4247

Éditeur

Université Aix-Marseille (AMU)

Référence électronique

Jean-Michel Denizart, « La marge de manœuvre interprétative chez le cinéaste David Lynch : de Lost Highway à Inland Empire », Les chantiers de la création [En ligne], 7 | 2014, mis en ligne le 01 septembre 2014, consulté le 08 avril 2020. URL : http://journals.openedition.org/lcc/653 ; DOI : https://doi.org/ $10.4000 /$ lcc. 653

Ce document a été généré automatiquement le 8 avril 2020.

Tous droits réservés 


\title{
La marge de manœuvre
} interprétative chez le cinéaste David Lynch : de Lost Highway à Inland Empire

\author{
Jean-Michel Denizart
}

1 Il serait relativement aisé de décrire le cinéma de David Lynch comme cinéma de la marge, dans la mesure où l'ensemble des analyses textuelles dont il fait l'objet, et qui ont déjà fait couler beaucoup d'encre, lui reconnaissent une importante forme de rupture avec le cinéma dit "conventionnel », notamment de par la complexité à en saisir les structures. On pensera par exemple à la notion de "cinéma cerveau », décrite par Gilles Deleuze au sujet de Stanley Kubrick qui a été, mainte fois transposée à l'univers lynchien, tant la mécanique huilée de l'orange a pu ne serait-ce qu'inspirer Lynch et son cinéma de velours aux ramifications souterraines. Toutefois, ce dernier n'a rien d'un «bleu » et endosse pleinement son rôle d'artiste qui, comme le rappelle Michel Ciment ${ }^{1}$ citant Hegel, « se trouve à mi-chemin entre l'intellect et la sensibilité »; car c'est bien de cela qu'il s'agit chez Lynch: articuler un cinéma qui résiste, non comme soubassement d'une confusion gratuite, mais comme conséquence d'une poésie de la sensation pure et du mystère.

2 Cependant, et c'est le paradoxe que souligne Diane Arnaud, le cinéma de Lynch raconte, et qui plus est, des histoires somme toute banales, déjà maintes fois éprouvées, parmi lesquelles : « la difficulté qu'ont les hommes à choisir entre la brune, et la blonde, [...] des histoires de jalousie obsessionnelle et de tromperie, enfin l'attente d'une femme pour un rôle" (Arnaud). Différentes intrigues, majoritairement entendues et qui pourtant laissent systématiquement libre cours à une importante marge de manœuvre interprétative, à l'intérieur de laquelle s'engouffre notamment une foule d' « interméneutes ${ }^{2} »-$ pour reprendre l'expression d'Hervé Aubron - qui se livrent, en ligne, à de véritables joutes de sens. 
3 Pour tenter de résoudre cette apparente dichotomie, nous proposons d'interroger le Lynchland, non plus comme territoire cinématographique excentré, mais davantage comme double espace de création (au sens d'une co-construction de sens du réalisateur d'une part et du spectateur d'autre part) ; car comme nous l'introduisions, le cinéma narratif de Lynch raconte... reste encore à en déterminer les modalités.

4 À l'aune de la théorie sémio-pragmatique de Roger Odin - qui nous servira de garant théorique -, et plus ponctuellement de la sémiotique de Peirce, nous nous efforcerons dans un premier temps de définir l'espace de communication dans lequel nous délimitons notre réflexion. C'est-à-dire de sélectionner les contraintes qui régissent le processus de production de sens en fonction de l'axe de pertinence que nous choisirons pour notre analyse.

5 Une fois établie, cette conjoncture - dont la vocation est également d'introduire les premières bases d'un raisonnement par étapes qu'il faudra par la suite approfondir nous verrons comment certains processus et opérations qui composent le mode de lecture "fictionnalisant ", c'est à dire celui qui me conduit à "vibrer au rythme des événements fictifs racontés ${ }^{3}$, sont par moment difficiles à appliquer au système de vibration de Lynch, tant sa volonté de figurer l'impossible abstraction (ce qui n'est pas sans répercussions sur la configuration espace/temps du récit) semble y jouer un rôle prépondérant. En résulte, une certaine forme de marginalisation du spectateur qui fait l'expérience de son relatif abandon aux pouvoirs d'une figuration nouvelle, car Lynch ne renonce à rien. Il «tire des possibilités du cinéma le maximum, quitte à exagérer les tensions, les discontinuités, les rapts sensoriels, les chevauchements ...», ce qui désarçonne le spectateur, «peu préparé à un tel déploiement d'énergie, à une telle combinaison de forces, qui n'ont pas le sens pour cible - encore moins pour source » (Astic, 23). Il conviendra alors de se demander quels rapports entretient la marge de manœuvre que nous considérons avec cette dispersion du sens qui s'instaure à l'intérieur d'un territoire interprétatif dont l'apparente absence de frontière semble participer du reclassement de la notion de marge à celle de gouffre.

\section{La sémio-pragmatique comme cadre théorique}

\subsection{De la sémio-pragmatique}

6 La théorie sémio-pragmatique se revendique comme un modèle heuristique ${ }^{4}$ de communication ou du moins de «non-communication» dont l'un des principes fondateurs affirme qu' "il n'y a jamais transmission d'un texte d'un émetteur à un récepteur, mais un double processus de production textuelle : l'un dans l'espace de la réalisation et l'autre dans l'espace de la lecture » (Odin, 2000, 10). Autrement dit, considérer la transmission d'un même texte (T) de l'actant émetteur (E) vers l'actant récepteur $(\mathrm{R})$ ne serait qu'illusoire dans la mesure où dans l'espace de lecture, $(\mathrm{T})$ n'existe plus sous la forme d'une entité textuelle signifiante, mais comme une somme de flux à partir desquels $(\mathrm{R})$ (re)construit un texte $\left(\mathrm{T}^{\prime}\right)$. La théorie sémio-pragmatique invite donc à s'interroger sur la manière dont se construisent les textes et sur les conséquences de telles constructions, mais aussi à se demander comment une fois investis dans leur espace respectif, les processus de production textuelle et les différentes contraintes qui pèsent sur eux, peuvent donner naissance à deux entités $(\mathrm{T})$ 
et ( $\left.\mathrm{T}^{\prime}\right)$ dont l'apparente similitude participe de l'illusion d'une translation de (T) de l'espace de réalisation vers l'espace de lecture.

7 Roger Odin formule également l'hypothèse que toute production textuelle est décomposable en une somme limitée de modes de production de sens et d'affects et dont l'ensemble forme notre «compétence communicative ». Après plusieurs ouvrages, il parvient à recenser un peu moins d'une dizaine de modes, eux-mêmes décomposables en une somme de processus et d'opérations.

Puisque l'une de nos finalités est bien de déterminer les modalités du cinéma narratif de David Lynch, nous initierons notre démarche à partir du mode "fictionnalisant ", qui se décrit comme «le mode qui me conduit à vibrer au rythme des événements fictifs racontés $»^{6}$, et dont nous retiendrons plus particulièrement les deux premiers processus constitutifs, à savoir :

- La diégétisation ${ }^{7}$ (niveau de l'espace) ou construction d'un monde.

- La narrativisation (niveau discursif) ou construction d'un récit.

9 A partir de là, nous formulons l'hypothèse que s'interroger sur la «marge de manœuvre interprétative » dont dispose le spectateur spécifiquement à l'intérieur du territoire narratif Lynchien, c'est, au regard des contraintes considérées, questionner les ressemblances et les dissimilitudes existantes entre l'orientation narrative $\left(\mathrm{N}_{\mathrm{T}}\right)$ conférée par le réalisateur au texte $(\mathrm{T})$, et son interprétation $\left(\mathrm{N}_{\mathrm{T}}{ }^{ }\right)$, qui s'instaure au sein d'une opération plus vaste de (re)construction par le récepteur du texte ( $\left.T^{\prime}\right)$. Toutefois, questionner la "marge de manœuvre interprétative ", c'est également comparer les différents $\left(\mathrm{N}_{\mathrm{T}}{ }^{\prime}\right)$ - que l'on dénommera $\left(\mathrm{N}_{\mathrm{T}}{ }^{\prime}\right)_{1},\left(\mathrm{~N}_{\mathrm{T}}{ }^{\prime}\right)_{2},\left(\mathrm{~N}_{\mathrm{T}}{ }^{\prime}\right)_{3}$, etc. interprété par chaque spectateur.

À l'inverse des conceptions les plus radicales du courant constructiviste, qui considère qu'il y a autant d'interprétations possibles que subsistent d'interprètes et d'interprétants ${ }^{8}$, envisager une marge de manœuvre interprétative théoriquement nulle, reviendrait alors à supposer que l'ensemble des entités narratives construites puissent êtres identiques. À contrario, plus la marge de manœuvre est importante, moins les entités $\left(\mathrm{N}_{\mathrm{T}}\right),\left(\mathrm{N}_{\mathrm{T}}{ }^{\prime}\right)_{1},\left(\mathrm{~N}_{\mathrm{T}}{ }^{\prime}\right)_{2}$, etc. seraient semblables. Cependant, reste encore à fixer les contraintes qui régissent de tels processus de constructions avant de pouvoir se lancer dans leur analyse.

\subsection{Des contraintes sélectionnées à l'« espace de communication » considéré}

11 Le courant épistémologique dans lequel nous inscrivons notre réflexion tente d'expliciter les relations entre le film et le spectateur dans une situation de projection où la dimension pragmatique des circonstances de l'interprétation est fondamentale. Si la notion de contexte se laisse définir comme un faisceau de contraintes influant sur le déroulement de la situation de communication considérée, il est alors nécessaire de le délimiter. Une tâche pour le moins complexe si l'on envisage le fait que ces contraintes sont extrêmement nombreuses. François Latraverse, cité par Roder Odin, y voit un "problème théoriquement et philosophiquement intéressant, encore, ajoute-t-il, qu'il soit parfois quelque peu désespérant » (Odin, 2011, 25).

12 La notion d'« espace de communication $»^{9}$ propose au moins une alternative, sinon une échappatoire en tentant de « modéliser » ce faisceau de contraintes. Il s'agit donc d'une 
construction du théoricien, au sens que l'« espace de communication », à la différence du contexte, n'a rien de concret, mais que sa conception passe par le choix d'un axe de pertinence orientant l'analyse et permettant de circonscrire un ensemble de paramètres maîtrisables, c'est-à-dire de sélectionner un nombre limité de contraintes régissant le processus de production de sens. L'essentiel étant ici d'afficher clairement nos limitations, comme Christian Metz le conseilla souvent à Roger Odin :» il suffit de le dire » (41).

13 L'espace de communication que nous avons construit et analyserons dans la suite de notre développement se veut volontairement simple et circonscrit, ceci afin de mieux se prêter à l'exercice de l'article et de ses ambitions introductives, sans quoi nous risquerions de basculer dans un véritable travail de thèse. Nous n'en restons pas moins cependant convaincus que même s'il s'agit d'une modélisation relativement restrictive, ce cas de figure reste toutefois représentatif de bons nombres de configurations et malgré son apparente simplicité, il est tout à fait pertinent de s'efforcer d'en comprendre toutes les ramifications avant d'étudier une quelconque situation de communication dans toute sa complexité, c'est-à-dire aux contraintes plus nombreuses et/ou superposant différents espaces.

Notre espace considère donc la situation classique d'un spectateur se rendant au cinéma afin d'assister à la projection d'un des films qui compose notre corpus, c'est à dire : un dispositif cinématographique (ou opérateur de communication) à l'intérieur duquel l'actant $\mathrm{E}$ (émetteur) est positionné en tant que réalisateur, et l'actant $\mathrm{R}$ (récepteur) en tant que spectateur. Un spectateur solitaire dont le poids du dispositif participerait de sa relative exclusion du reste de la salle, qui serait confronté par choix personnel à l'un de ces trois films de David Lynch qu'il n'a encore jamais vu : Lost Highway (1997), Mulholland Drive (2001) ou Inland Empire (2006), dont l'implication serait totale (Esquenazi) et qui ne ferait l'objet d'aucune consigne de lecture particulière, développant ainsi une axe de pertinence issu du film lui même, mais aussi des contraintes externes ${ }^{10}$, suggérant fortement une lecture de type fictionnalisante. Nous intitulerons cet espace: "Espace de communication cinématographique lynchien dit “classique", de 1997 à nos jours ".

Comme nous l'introduisions plus haut, les contextes et leurs contraintes sous-jacentes sont nombreux. Ainsi, le dispositif en constitue une première catégorie qui dans le cadre cinématographique, s'avère particulièrement stable et injonctif. Les cadres social et contextuel communicationnel qui précèdent la lecture des données et orientent les significations considèrent respectivement quant à eux, la dimension sociale du choix du film ainsi que les consignes de lecture contextuelle que le spectateur est normalement libre ou non de respecter (dans notre cas, le spectateur de fiction à l'«implication totale » s'efforcera de percer l'histoire racontée par le film qu'il a choisi et de rester attentif). Si ces types de contextes demeurent en temps normal, davantage mouvants et diachroniques, le but de notre espace est d'en «fixer artificiellement » le faisceau de contraintes, et, sans pour autant occulter les variables précédemment citées, mieux redéployer notre analyse sur le "contexte réactionnel ${ }^{11}$ construit au cours de la diffusion, délibérément laissé mouvant et qui, nous le pensons, sera le plus à même d'offrir de premiers éléments de réponse à nos interrogations sur la marge de manœuvre interprétative.

Le contexte réactionnel se laisse décrire comme un «événement mouvant et évolutif par excellence, compte tenu du rythme de renouvellement des données (filmiques) et 
des différents interprétants mobilisés par le spectateur, le contexte d'aperception change et se modifie au gré de l'avancée du film et de l'interprétation du spectateur ${ }^{12}$.

Enfin, le choix de ce corpus filmique qui compose notre système n'a également rien d'anodin. Si nous excluons The Straight Story («Une histoire vraie », 1999), qui s'avère pourtant symptomatique de la capacité de David Lynch à surprendre son public - mais davantage ici grâce à une polyvalence que peu lui connaissait, qu'au travers des procédés filmiques qu'il utilise - Lost Highway marque un véritable tournant dans la filmographie du cinéaste et préfigure par bien des aspects Mulholand Drive et Inland Empire. Hervé Aubron reconnaît d'ailleurs la raréfaction depuis Twin Peaks, Fire Walk With Me (1992) des "scènes boites", c'est-à-dire les séquences conçues «comme des cabarets ou des tableaux circonscrits à l'intérieur des films». De véritables « installations autarciques [...] qui se créaient une estrade propre et aussi un espace temps-particulier, de faible profondeur: une frontalité presque bidimensionnelle, archaïque et "égyptienne", ainsi que l'a justement écrit Michel Chion » (Aubron, 2006, 76).

Si de telles séquences sont bien évidemment toujours présentes, l'aporie dont témoigne le cinéma Lynchien ne se manifeste plus aujourd'hui que exclusivement dans l'exhibition de scénettes à la fois marginales et sidérantes ${ }^{13}$, mais vient contaminer l'ensemble d'une narration dont l'altération la plus évocatrice se concrétise par l'intermédiaire d'une rupture totale de la trame, scindant ainsi chacun de ses trois films en deux parties distinctes.

\section{Un cinéma qui favorise l'abstraction : La trichotomie lynchienne}

19 Comme nous l'introduisions, le premier processus constitutif de la fictionnalisation s'intitule la diégétisation. Ce processus se subdivise lui même en différentes opérations, dont la première d'entre elle : la figurativisation. Par «figuratif », Roder Odin, entend comme Algirdas Julien Greimas: " un contenu qui a un correspondant au niveau de l'expression de la sémiotique naturelle $\aleph^{14}$, ce qui revient à dire qu'outre toute consigne de lecture particulière, il est impossible de diégétiser à partir d'un support considéré comme abstrait.

Or, à l'intérieur de notre corpus, nous relevons que David Lynch ose le pari impossible de "figurer l'abstraction », du moins de grandement la favoriser, en poussant dans ses retranchements les limites de la figurativité à travers le développement de différentes ambiances qui proposent un retour aux sensations brutes, aux émotions pures, à la perception première - ou oserions-nous dire, en référence à la triade Peircienne, «primaire »- du monde. En ce sens, le cinéaste rejoint encore une fois les considérations de Stanley Kubrick, pour qui « un film est - ou devrait être - beaucoup plus proche de la musique que du roman. Il doit être une suite de sentiments et d'atmosphères » (Stanley Kubrick, 1964).

21 Dans cette même logique, il n'est pas étonnant de se confronter au sein de notre échantillon filmographique, à la volonté Lynchienne de matérialiser trois qualités ou pures potentialités abstraites à la fois particulières et récurrentes, que sont : l'absence, la simultanéité et le sensible (ou psyché de ses personnages). 


\subsection{L'absence} disparition, par l'intermédiaire des relations qu'elles entretiennent respectivement avec la trace et le vestige; ce qui revient à signifier le manque par le biais de symptômes qui au sein de notre corpus sont légion. Dans le cadre de Lost Highway, Guy Astic fait allusion à la volonté du cinéaste de faire transparaître la "face cachée " de ses séquences à travers "les sautes d'humeurs immotivées", "les éclats intempestifs du filmage ", "l'implicite des dialogues » qui se veulent, dans leurs rares manifestations, relativement laconiques (3312 mots prononcés dans tout le film) ou enfin « l'insistance de la caméra sur l'inerte " (Astic, 18) - on pensera à l'aménagement spatial pour le moins spartiate de l'intérieur de la maison des Madison.

Dans un rapport plus axé autour de la question du temps, l'absence se matérialise également dans l'obsolescence mémorielle de protagonistes qui font parfois jusqu'à l'expérimentation d'une amnésie la plus totale, mais aussi d'importants troubles de la personnalité. Démunis face à leur incapacité à se remémorer certains événements déterminants - qui font par conséquent aussi bien défaut aux personnages qu'au spectateur -, ces derniers sont également tourmentés par différents souvenirs dont le caractère réminiscent nous invite constamment à en remettre en question la légitimité. Qu'il s'agisse de la «métempsychose $»^{15}$ dont souffrent chacun des protagonistes principaux des trois films, du passé envolé de Rita, de cette fameuse nuit, lourde d'un profond secret que les Dayton ne sauraient révéler à leur fils ou de la transformation conjointe d'un Fred Madison plongé dans l'incertitude d'avoir oui ou non tué sa femme.

\subsection{La simultanéité/superposition}

Meurtres dans Lost Highway, qui, plus manifestement avec la mort de Dick Laurent, viennent d'ailleurs profondément alourdir l'atmosphère de début de film. Si, dans le temps de la projection, leurs manifestations sont chronologiquement postérieures aux scènes d'ouvertures, la volonté auctoriale d'également figurer simultanéité et superposition, si elle rend complexe l'attribution d'un temps, mais aussi d'un espace narratif, permet néanmoins au cinéaste d'imprégner certains segments filmiques de conséquences relatives à des événements encore non survenus mais aussi de superposer différents interstices. Si l'on reconnaît certains symptômes de la crise image-action introduite par Gilles Deleuze et que l'on sait chère à David Lynch, ce dernier va plus loin en ôtant toute pertinence aux notions de passé, futur et frontières qui tendent alors inexorablement à se chevaucher. "Le lointain et le contigu se confondent, le distant et le proche ne se distinguent plus réellement » (37).

On pensera dans un premier temps aux boucles que viennent boucler le fameux « Dick Laurent is dead " dans Lost Highway; l'ultime "This is the girl " dans Mulholland Drive, accompagné de sa mystérieuse clef bleue, qui en guise de conclusion et de manière très analogue à la mort de Renee et Dick Laurent préfigurent un double meurtre dont l'initiative - loin de toute logique de flashback - est pourtant à l'origine même de la trame narrative; ou enfin le personnage crédité «la fille perdue» («Lost Girl»), interprété par Karolina Gruszka dans Inland Empire.

Dans un second registre, il est également aisé de citer la première rencontre entre Fred Madison et l'Homme Mystère aux talents ubiquitaires, la fusion entre l'étage de la villa

Les chantiers de la création, 7 | 2014 
d'Andy et les chambres de l'hôtel Lost Highway, les prémonitions oniriques de Diane Selwyn quant à son suicide, ou encore la frontière poreuse entre les univers de Nikki Grace et Susan Blue, c'est-à-dire entre le film et le film dans le film, à la manière d'une hétérotopie ${ }^{16}$ en forme de poupée russe.

\subsection{Le sensible (ou psyché des personnages)}

Cette altération spatio-temporelle trouve également ses origines dans le fait que depuis Lost Highway, David Lynch tente désormais d'exprimer « des mondes de plus en plus intérieurs dans lesquels ses personnages se retranchent, confrontés à leurs déceptions, leurs peurs et leurs gestes extrêmes » (Lynch, Rodley, 7). L'espace diégétique Lynchien, semble en effet corrompu par la projection d'états de conscience instables traversant ses protagonistes, par exemple le fantasme, la jalousie, l'hallucination, le cauchemar, etc. Si le cinéaste multiplie ainsi les débordements de la vie intérieure, au point qu'au même titre que les frontières physiques, intériorité et extériorité tendent à fusionner, «il ne le fait pas que par unique intérêt pour l'aberration psychique. Il travaille à hauteur de l'aberration filmique, déverrouille ainsi les modes ordinaires de perception, oblige à voir l'irreprésentable (Astic, 55). On peut ainsi dire que l'image schizophrénique rive donc les personnages (et le régime filmique) aux marges du temps et de l'espace, les fait évoluer à la fois en dedans et en dehors » (59).

28 Si nous n'y faisions qu'allusion un peu plus tôt, nous pensons évidemment au cas de Pete Dayton qui vient malencontreusement d'abattre Andy dans sa villa, puis de découvrir une photo exhibant Renee Madison et Alice Wakefield. Pete semble alors accuser le coup de cette révélation, à la fois physiquement - les saignements de nez -, mais aussi mentalement. Ce trouble psychique prend la forme d'un "étirement anamorphique » au sein duquel le jeune Dayton montant à l'étage est confronté à Renee (à moins que ce ne soit Alice) et Dick Laurent, dans la chambre vingt-six de l'hotel Lost Highway. Les éclats filmiques correspondent à l'«avant-première stroboscopique " (Arnaud) d'une scène qui n'aura lieu que plus tard. Le spectateur est alors incapable de conceptualiser cette contraction spatiale et temporelle, il est bercé par l'inquiétante étrangeté de partager à hauteur diégétique, l'hallucination dont fait l'objet Pete.

De manière analogue, chaque partie de Mulholland Drive pourrait s'avérer la réciproque rêvée de sa composante voisine qui deviendrait alors la réalité. Les deux entités interagissant tout au long du film et menaçant à chaque instant de se révéler mutuellement, la volonté de les discriminer dans une approche strictement onirique semble par conséquent manquer de pertinence.

Enfin, nous pouvons encore une fois citer le cas de Nikki Grace, interprétant le rôle de Susan Blue, le personnage principal d'une intrigue adultérine, qui, à partir de l'instant où Nikki se rapproche de Davon Berk, alias Billy Side, sombre peu à peu dans une épopée violente et déchirante. Inland Empire apparaît alors comme une forme de «metafilm fantastique » qui tente de nous faire vivre cette sensation de ne plus être vraiment soi à travers l'ambivalence de Nikki qui «comme sous l'impulsion d'une forme à la fois punitive et libératrice, avait traversé le miroir et se retrouvait enfermée dans la vie de son personnage » (Arnaud). 


\section{Conséquences narratives}

\subsection{Diégétisation complexe}

31 Si comme nous venons de le voir, l'univers figural de David Lynch, de par sa volonté abstractionniste, tend intrinsèquement à entrer en conflit avec la première opération de figurativisation, elle n'est pas la seule étape du processus de diégétisation à souffrir de ce que nous avons dénommé la « trichotomie lynchienne ». L'ultime opération de ce processus, qui «se fonde sur la potentialité narrative que je reconnais à un espace $»^{17}$, c'est-à-dire sur ma faculté à considérer celui-ci comme habitable, par un personnage ou par moi même, est également profondément affectée ${ }^{18}$.

Cette opération n'est pas d'ailleurs sans rappeler les propos d'Augustin Fressin, qui dans son analyse croisée des travaux de Bergson et Merleau-Ponty, attribue au regard le devoir ainsi que le pouvoir d'instituer l'espace environnant l'objet de ma perception, comme « lieu possible de mon action » (Fressin, 63) ; or, comme le suggère Paul Ricoeur dans Temps et récit, toute action peut être vue en tant que potentialité narrative ${ }^{19}$.

Le Lynchland esquisse les marges d'une diégèse titubante qui ne remplit pas les conditions nécessaires d'une localisation univoque et parachevée. Elle arbore tout au plus différentes lignes de force, à la fois saillantes et éphémères, dont le spectateur est chargé d'assurer la topologie, à la manière d'un vaste complexe architectural aux délimitations conditionnelles et aux composantes très souvent difficiles à articuler entre elles. Un espace évanescent, que les personnages, dans toute l'expression de leur précarité, ne font au mieux qu'arpenter, laissant ainsi traîner derrière eux de vastes sillons que le récepteur, dans sa volonté diégétisante, a bien du mal à choisir ou à emprunter. Nous pourrions ainsi citer l'intérieur labyrinthique des Madison ; la maison sur pilotis dont la suspension est à l'image de l'aspect conjectural sur lequel reposent le bungalow ainsi que les protagonistes qui y croisent leurs identités ; l'hôtel Lost Highway, lieu de passage par excellence aux frontières perméables; l'aquarium inhospitalier dans lequel est exhibé Mr. Roque mais qu'on oserait trop tenter de localiser; les manifestations intempestives de l'univers de la série Rabbits, l'indissociabilité des mondes respectifs de Susan, Nikki et la «fille perdue »; etc.

De plus, comme nous l'évoquions, pénétrer dans un des films de notre corpus, c'est aussi composer avec l'intériorité à la fois débordante et contagieuse de protagonistes dont l'environnement n'a parfois que pour seule vocation d'assurer la continuité de leurs états de conscience.

L'altération de la figurativisation et de notre faculté à reconnaître un espace fictionnel comme habitable concourent ainsi à ouvrir une déchirure dans laquelle il est difficile de se projeter et de construire un récit.

\subsection{La partie pour le tout}

«La perception nous fait assister à ce miracle d'une totalité qui dépasse ce qu'on croit être ses conditions ou ses parties » (Merleau-Ponty, 23).

$\mathrm{Si}$, à la manière de Merleau-Ponty, David Lynch reconnaît la prégnance de la totalité signifiante cinématographique sur la somme de ses parties, il ne cache cependant pas son attirance de la partie vers le tout : "Je trouve le fragment très intéressant. On peut rêver le reste. C'est alors que vous participez " (Lynch, Rodley, 21). Tout comme la 
définition que Fressin donne à l'objet dans sa relation au sujet, Lynch ne nous fait volontairement entrevoir qu' « un minimum du perceptible (Fressin, 63) qui sert de soubassement à un cinéma "panique ». Et c'est également bien en cela que réside la figuration de l'absence : la réclusion extraordinaire des conditions de compréhension qui n'a ni plus ni moins pour conséquence l'effondrement de la totalité, sous le poids de la déficience de liens structurants. Parmi ces modalités manquantes, nous pensons à la difficulté que nous avons de constituer et d'organiser un ensemble de catégories sémantiques qui « rend possible la lecture uniforme du discours » (Greimas, J. Courtés, 197).

\subsection{Au niveau discursif}

37 La citation précédence, fait référence à la définition que donne A. J. Greimas du principe d'« isotopie ", qui doit son origine à la branche sémantique de la linguistique, et dont l'étude permet d'accéder au sens du texte, tel que celui-ci a été pensé par son auteur.

D'un point de vue discursif, la construction d'un récit passe par différentes étapes dont la constitution d'une isotopie sémantique, sa structuration temporelle et paradigmatique permettent dans un premier temps de sélectionner et d'agencer «ce qui est pertinent pour l'avancée du récit par rapport aux indices de vraisemblance ou aux digressions" (Odin, 2000, 30). Cette première construction, comme le démontre Umberto Eco cité par Odin, est motivée par une isotopie de lecture dont le choix est initié à partir d'une hypothèse thématique (ou topic) liée au spectateur lui-même ainsi qu'aux contraintes externes. Cette notion de topic peut être rapprochée de l'intentionnalité première (degré zéro) résultante des contextes précédant la projection et dont nous avons volontairement fixé et généralisé qu'ils induisaient une lecture fictionnalisante. Cependant, en plus de ce faisceau de contraintes, nous considérons bien évidemment le contexte réactionnel à l'intérieur duquel cet ensemble d'opérations, que nous rapprocherons d'une structuration de successions d'unités de sens ${ }^{20}$ est également fonction de l'expérience du spectateur, du contexte narratif, c'està-dire de ce qui a été vu et entendu au cours de la projection, et donc des interprétants et des intentionnalités nouvelles que le récepteur parvient à mobiliser.

\subsection{De la narrativisation vers la narration}

C'est ainsi que tout public, dans sa volonté fictionnalisante, est amené à construire en arrière-plan différentes unités de sens autonomes - que l'on qualifiera de narrativisantes - qui appartiennent à un niveau de "pré-compréhension " (29), puis, dans un double mouvement à la fois prospectif d'horizon d'attente et rétroactif de restructuration des acquis, contextualiser toutes ces unités à travers leurs relations sémantiques, syntagmatiques, paradigmatiques et causales. Ceci, afin d'accéder en fin de processus, à un méta-niveau propre à une narration pleine dont l'apogée se concrétise après la prise de connaissance de la conclusion filmique, par la production d'une grande transformation reliant la fin au début du récit, car comme le dit Ricoeur cité par Odin : "Comprendre l'histoire, c'est comprendre comment et pourquoi les épisodes successifs ont conduit à cette conclusion, laquelle, loin d'être prévisible doit être finalement acceptable comme congruente avec les épisodes rassemblés » (31). 
Le trouble qui mine les films de notre corpus est relatif à la dualité expériencée par le spectateur qui, après avoir matérialisé les nombreux régimes d'actions perçus en autant d'unités de sens circonscrites, est déchiré entre son incapacité à les sélectionner puis les articuler entre elles et l'attente de nouveaux éléments de compréhension déterminants qui ne se manifesteront finalement pas, du moins pas aussi explicitement qu'espéré. En clair, il est donc complexe de passer de la narrativisation à la narration.

\subsection{Une logique générale du mystère}

"C'est comme ça que ça doit être; il ne s'agit pas de confusion, il faut ressentir le mystère. Le mystère, c'est bien, la confusion, c'est mauvais; il y a une grande différence entre les deux » (Lynch, Rodley, 173).

Pénétrer dans notre corpus, c'est littéralement assister à une projection qui aurait déjà commencé en notre absence, à une histoire bien entamée, hantée par les conséquences d'événements au conditionnel et dont nous ne savons réellement si ces derniers sont en devenir ou s'ils ont déjà eu lieu, mais dont les vestiges transparaissent dans l'incapacité que nous éprouvons à contextualiser, à ancrer les données qui nous sont proposées. Si nous évoquions un peu plus tôt les morts de Renee, Dick Laurent, Camilla et Diane, qui illustrent de manière fantastique ce sentiment de film entamé dans la mesure où leurs conséquences préfigurent leurs avènements, elles ne sont pas les seules. La première partie de Lost Highway est particulièrement évocatrice de ce phénomène, en ce qu'elle prolonge la sensation qu'on éprouve en commençant un film; c'est-à-dire une importante accumulation de premières questions qui d'ordinaire trouverait réponse rapidement, mais qui avec Lynch vous hante sans que vous ne vous en rendiez vraiment compte, depuis déjà quarante minutes. Que penser des rapports qu'entretiennent Renee et Fred ? Leur relation semble assombrie par certaines difficultés. L'impuissance sexuelle suggérée du mari est-elle à l'origine de l'absence d'un enfant au sein du couple? (Absence à laquelle les questions indiscrètes des détectives et l'air embarrassé de Renee pourraient également renvoyer : "Il y a-t-il une autre chambre»?). Cette incapacité à satisfaire sa femme et les désirs du couple a-t-elle enjoint Fred à développer parallèlement une certaine forme de jalousie obsessionnelle, par peur d'être trompé ? Ou bien Renee est-elle vraiment indigne de confiance ? Mais qui est-elle vraiment? Quelle est sa véritable histoire ? ( $C$ C'était il y a longtemps, et c'est là que tout a commencé... »), etc. On pourrait être en droit d'imaginer que les réponses à ces questions se trouvent dans un « avant-film » qui nous aurait été caché tant la suite des événements continue de nous plonger dans l'incertitude.

« Le tout peut avoir une logique, mais en dehors de son contexte, le fragment prend une incroyable valeur d'abstraction. » (Lynch, Rodley, 174).

Le réalisateur nous prive délibérément du contexte d'interprétation, ce qui a pour effet d'imposer au récepteur d'opérer de réguliers et massifs renouvellements d'unités de sens narrativisantes qui par l'absence d'ancrage contextuel, tendent à rester circonscrites.

Comme l'introduisait David Lynch : « c'est alors que vous participez ». Le spectateur est à l'évidence grandement sollicité. Il doit « redéployer et connecter ce que le film tend à contracter, superposer et doubler» (Astic, 56), c'est-à-dire abandonner, au moins partiellement, tout modèle du récit qu'il aura pu acquérir, ainsi que l'idée qu'il puisse exclusivement se reposer sur ses propres connaissances collatérales filmiques, afin de s'ouvrir à de nouvelles formes de perception et de compréhension organiques. S'il peut 
arriver au sein de films «traditionnels» que le spectateur ne parvienne pleinement à saisir certaines unités de sens dont l'articulation ferait appel à des acquis personnels qui lui feraient défaut, traverser le Lynchland est un exercice qui s'avérera systématiquement problématique en ce qu'il exige des compétences particulières, en ce qu'il exige de dépasser la matière à la fois éparse et lacunaire qui nous est proposée. Ajoutez à cela que le récepteur doit tisser son chemin au travers de séquences présentant chacune une intensité particulière, lui faisant croire que le scénario s'apprête à adopter une nouvelle direction qui à peine empruntée implosera aussitôt, " comme si la trame était prise dans un mouvement de tissage et de décrochage sans cesse réactivé » (18).

Un chemin dont les relations qu'il aspire à mettre en relief n'ont par conséquent rien de clairement balisé et que l'abstraction ambiante, la diégèse vacillante et les débordements psychiques des protagonistes tendent à obscurcir. Toute poussée significationnelle, toute construction, tout agencement d'unité est inférée par le spectateur au travers d'une démarche, qu'en référence à Charles Sanders Peirce nous qualifierons d' "abductive", en ce qu'elle met en œuvre différentes hypothèses, spéculations, approximations successives ou tout au plus des intuitions, mais dont on ne sait jamais si elles aboutiront à percer le sens profond du film, car rien ne nous amène jamais à les confirmer.

Même en adoptant d'hypothétiques clefs de lectures que la communauté Lynchienne est parvenue à faire émerger parmi d'autres, après de nombreuses analyses a posteriori de notre corpus - qu'il s'agisse des théories de la fugue psychogénique de Fred Madison, du rêve de Diane Selwyn inspiré d'éléments de sa véritable vie, de la revanche de la jeune Polonaise perdue sur ses démons passés, ou de la construction narrative des films en forme de ruban de Mobius -, «l'énigme n'est jamais vraiment refermée, les sens jamais comblés, les émotions jamais expurgées » (23).

\section{Conclusion}

«Les images du film sont les mêmes - toujours de la même durée, la même bandeson. Mais l'expérience dans la salle est différente selon le public. C'est une autre raison pour ne pas trop en dire aux spectateurs, parce que le "savoir" putréfie cette expérience " (Lynch, Rodley, 214).

Par «ne pas trop en dire », il faut entendre que David Lynch, par amour de l'abstraction et du fragment, plonge délibérément le récepteur dans un contexte narratif tronqué. Ce qui rend ce dernier incapable de finaliser son raisonnement, ou, pour le dire en termes sémiotiques: incapable de convoquer un interprétant logique final, laissant ainsi ouvertes les multiples pistes que le spectateur détective, dans la démarche abductive que nous évoquions, avait empruntées. Toutes ces pistes sont autant d'« expériences » différentes et potentielles, autant de reconstitutions $\left(\mathrm{N}_{\mathrm{T}}\right)$ ' de $\left(\mathrm{N}_{\mathrm{T}}\right)$ qui, faute de pouvoir être fermement sélectionnées, coexistent, cohabitent.

Au regard des éléments que nous avons été capables de mettre en relief, et dans le cadre de notre réflexion sur la marge de manœuvre interprétative, il paraît relativement difficile, voire dangereux d'affirmer que chaque spectateur développera exactement le même ensemble d'hypothèses - auquel cas, bien que les interprétations soient plurielles, la marge pourrait être considérée comme réduite, chaque spectateur aboutissant au même résultat : 


$$
\left(\mathrm{N}_{\mathrm{T}}\right)^{\prime}{ }_{\text {spectateur1 }},\left(\mathrm{N}_{\mathrm{T}}\right)^{\prime \prime}{ }_{\text {spectateur1 }},\left(\mathrm{N}_{\mathrm{T}}\right){ }^{\prime \prime}{ }_{\text {spectateur1 }} /\left(\mathrm{N}_{\mathrm{T}}\right)^{\prime}{ }_{\text {spectateur2 }},\left(\mathrm{N}_{\mathrm{T}}\right)^{\prime \prime}{ }_{\text {spectateur2 } 2},\left(\mathrm{~N}_{\mathrm{T}}\right){ }^{\prime \prime}
$$

ou qu'au contraire, tous se livreront à différentes adjonctions particulières de suppositions - la marge de manœuvre serait alors considérée comme importante, chaque spectateur aboutissant à un résultat différent :

$$
\begin{aligned}
& \left(\mathrm{N}_{\mathrm{T}}\right)^{\prime}{ }_{\text {spectateur1 }},\left(\mathrm{N}_{\mathrm{T}}\right)^{\prime \prime}{ }_{\text {spectateur1 }} /\left(\mathrm{N}_{\mathrm{T}}\right)^{\prime}{ }_{\text {spectateur2 }},\left(\mathrm{N}_{\mathrm{T}}\right)^{\prime \prime}{ }_{\text {spectateur2 }} / \\
& \left(\mathrm{N}_{\mathrm{T}}\right)_{\text {spectateur3 }},\left(\mathrm{N}_{\mathrm{T}}\right)^{\prime \prime}{ }_{\text {spectateur3 }},\left(\mathrm{N}_{\mathrm{T}}\right)^{\prime \prime \prime}{ }_{\text {spectateur3 } 3} \cdots
\end{aligned}
$$

Cependant, ce qui est alors certain, c'est qu'un même spectateur, et plus particulièrement le spectateur de la première fois que nous considérons, parviendra difficilement, face à notre corpus, à justifier une seule et même interprétation, à la fois ferme et univoque. En ce sens, lui est délibérément laissé le loisir de voguer d'une terre de compréhension à l'autre, tout en proscrivant la possibilité d'y trouver refuge, telle une pénitence au parfum d'exil. Toutefois, si nous pensons que les hypothèses de lectures sont clairement plurielles, leur nombre n'est pas pour autant infini, car les balises ou intrigues générales de Diane Arnaud, que nous évoquions en introduction, bien que sommaires, existent.

Qu'en est-il alors de la marge de manœuvre interprétative du cinéma de Lynch? Il convient pour conclure de se demander si le gouffre que feint de nous proposer le cinéaste ne correspond pas précisément aux limbes dans lesquels on a voulu nous plonger, tant ils parviennent à trouver une certaine logique. En effet, il ne nous reste qu'à accepter dans un premier temps, la figurativité comme principe organisateur du récit, ou, pour le dire cette fois-ci en termes sémio-pragmatiques, accepter une monstration qui tend par moment à s'autonomiser de la narration, pour servir l'ambition abstractionniste $\mathrm{du}$ processus de figurativisation. Ce qui revient à s'accommoder du risque de rompre avec l'opération de " mise en phase ${ }^{21}$ - qui exige que le travail de l'ensemble des paramètres filmiques soit au service du récit - et qu'il nous faille alors sanctionner ${ }^{22}$ notre expérience cinématographique par la manifestation d'une certaine frustration (ou pour les moins téméraires, d'un ennui profond). Car il est clair qu'à s'escrimer vainement à percer l'opacité du film, l'insatisfaction résultante peut s'avérer prégnante. A tel point que tous ceux qui ont eu la chance de s'entretenir directement avec l'investigateur d'un tel mystère, n'ont pu s'empêcher de l'interroger sur l'intentionnalité narrative $\left(\mathrm{N}_{\mathrm{T}}\right)$ qu'il a voulu insuffler à ses créations? C'est-à-dire sur leur sens véritable ? Encore faudrait-il qu'un tel absolu existe car le dernier élément avec lequel l'amateur du cinéma de Lynch sait composer, est bel et bien la fragmentation de la vérité.

\section{BIBLIOGRAPHIE}

Adjiman, Rémi, L'émergence du sens du spectateur, au cours de la projection cinématographique : une approche communicationnelle et cognitive, 2002.

-. Les variables audiovisuelles et la perception du spectateur, Aubagne : Cours dispensé au département SATIS de l'université d'Aix-Marseille, 2010. 
Arnaud, Diane, David Lynch - La double vie des personnages, http://www.cinematheque.fr/fr/danssalles/rencontres-conferences/espace-videos/david-lynch-double-vie-personnages,v,183.html, 2010.

Astic, Guy, Le purgatoire des sens: Lost highway de David Lynch, Paris: Dreamland, 2000.

Aubron, Hervé, Mulholland drive de David Lynch: dirt walk with me, Belgique: Yellow now, Crisnée, 2006.

Ciment, Michel, Le cinéma de Kubrick : entre raison et passion, http://www.cinematheque.fr/fr/ dans-salles/rencontres-conferences/espace-videos/cinema-kubrick-entre-raison-passion,v, 200.html, 2011.

Esquenazi, Jean-Pierre, Film, perception et mémoire, Paris : Ed. l'Harmattan, 1994.

Foucault, Michel, of Other Spaces, Heterotopias, Architecture, Mouvement, Continuité, Volume 5. 1894, pp. 46-49.

Fressin, Augustin, La Perception chez Bergson et chez Merleau-Ponty, Paris : Société d'édition d'enseignement supérieur, 1967.

Greimas, Algiras Julien, Courtés, Joseph, Sémiotique, dictionnaire raisonné de la théorie du langage, Paris : Hachette livre, 1993.

Lynch, David, Rodley, Chris, David Lynch : entretiens avec Chris Rodley, Paris : Cahiers du cinéma, 2004.

Merleau-Ponty, Maurice, Le visible et l'invisible, Paris : Gallimard, 1979.

Odin, Roger, De la fiction, Paris, Bruxelles : De Boeck Université, 2000.

-. La sémio-pragmatique : un modèle heuristique, Médias et cultures, n spécial. 2006, pp. 57-72.

-. Les espaces de communication : introduction à la sémio-pragmatique, Grenoble : Presses universitaires de Grenoble, 2011.

Ricoeur, Paul, La configuration dans le récit de fiction, Temps et récit, II, Paris : Seuil, 1984

\section{NOTES}

1. Ciment, M Le cinéma de Kubrick : entre raison et passion, http://www.cinematheque.fr/fr/danssalles/rencontres-conferences/espace-videos/cinema-kubrick-entre-raison-passion,v,200.html (2011).

2. Contraction d' " internautes » et " herméneutes » introduite par Hervé Aubron dans Mulholland drive de David Lynch : dirt walk with me (Belgique : Yellow now, Crisnée, 2006), p. 19.

3. Odin, R. De la fiction (Paris, Bruxelles : De Boeck Université, 2000), p. 11.

4. Odin, R. «La sémio-pragmatique : un modèle heuristique » (Médias et cultures, $n^{\circ}$ spécial. 2006), pp. 57-72.

5. Odin, R. De la fiction (Paris, Bruxelles : De Boeck Université, 2000), p. 11.

6. Ibid.

7. La construction d'une diégèse est une condition sine qua non à la narrativisation, en ce qu'elle fixe les «modalités de manifestation » (Odin, 2000, 22) de l'histoire. D'où l'attention particulière que nous lui accordons ici.

8. «L'interprétant peut être défini comme une norme sociale ou un habitus collectif, un « déjàlà » et la détermination ici et maintenant d'un esprit qui intériorise cette norme. L'interprétant doit être distingué de l'interprète, il est le moyen que ce dernier utilise pour effectuer son 
interprétation. Un interprétant est la modification d'une conscience causée immédiatement par le signe lors de la médiation vers l'objet. » (Adjiman, 2010, 18).

9. Odin, R. Les espaces de communication: introduction à la sémio-pragmatique (Grenoble: Presses universitaires de Grenoble, 2011), pp. 37-42.

10. Les contraintes externes tentent de « réduire la différence entre espace de l'émission et espace de la réception. [...] Pour tout ce qui concerne la communication de masse, c'est le rôle des genres qui servent à régler le système d'attente des destinataires en fixant l'axe de pertinence qui sera utilisé lors de la lecture. Raphaëlle Moine parle de "rails génériques" (2002). C'est aussi le rôle des grilles de programmes de télévision ». (Odin, 2011, p. 39).

11. On parlera également de « contexte narratif » dans le cas d'une lecture fictionnalisante. A ne pas confondre cependant avec la propre conception des « contraintes narratives » de Roger Odin. C'est pourquoi nous privilégierons la terminologie "réactionnel», ceci afin de lever toute ambiguïté.

12. Adjiman, R. L'émergence du sens du spectateur, au cours de la projection cinématographique: une approche communicationnelle et cognitive (2002), p. 410.

13. On pensera notamment à la virée nocturne de Frank dans Blue Velvet, qui se conclut par la prestation de Ben interprétant en play-back In Dreams, l'apéritif dans la cour du motel ou l'accident de voiture dans Wild at Heart, la Red Room de Twin Peaks, etc.

14. Odin, R. De la fiction (Paris, Bruxelles : De Boeck Université, 2000), p. 18.

15. La notion «métempsychose » ou «Doctrine de la transmigration des âmes" (TLF) est réutilisée par Hervé Aubron au sujet des multiples personnalités qu'adoptent dans Mulholland Drive, les protagonistes respectivement interprétées par Naomi Watts et Laura Harring. Qu'il s'agisse de Betty et Diane, de Camilla et Rita, ou de manière encore plus large des sentiments et orientations sexuelles nouvelles qui les traversent, de leurs métiers d'actrices qui outre la mise en abyme, sous-tendent par définition, l'incarnation de nouveaux rôles.

16. Foucault, M. "Of Other Spaces, Heterotopias ». Architecture, Mouvement, Continuité, Volume 5. 1894, pp. 46-49.

17. Odin, R. Ibid., p. 22.

18. Dans notre démarche, nous occultons pour l'instant volontairement l'opération d'« effacement du support » qui compose également le processus de "diégétisation » mais qui s'avère davantage en lien avec le dispositif cinématographique lui-même (dont nous avons reconnu le caractère injonctif) et sa matière de l'expression.

19. Ricoeur, P. «La configuration dans le récit de fiction », Temps et récit, II, Paris : Seuil, 1984.

20. Notre réflexion autour de l'unité de sens tient beaucoup du concept d'« unité filmique sémiocognitive ", qui se laisse notamment décrire comme "une entité de sens issue d'un travail de regroupement logique (au sens de la Gestalt) de l'interprétant, confrontée aux signes. Cette unité n'est pas univoque, elle contient en elle-même une grande complexité liée à la difficulté de la mise en sens et des processus d'institutionnalisation et de catégorisation » (Adjiman, 2002, 343).

21. Odin, R. De la fiction (Paris, Bruxelles : De Boeck Université, 2000), p. 37-46.

22. Odin, R. Les espaces de communication: introduction à la sémio-pragmatique (Grenoble: Presses universitaires de Grenoble, 2011), p. 40. 


\section{RÉSUMÉS}

Loin de proposer une approche traditionnelle de l'œuvre Lynchienne en tant que cinéma de la marge, notre analyse s'instaure dans la théorie sémio-pragmatique, pour - à partir de l'ensemble des outils que met à notre disposition ce modèle heuristique - tenter d'appréhender la marge de manœuvre interprétative dont font l'objet ses spectateurs, et ce, à travers une sélection de trois des films du grand cinéaste.

Far from offering a traditional approach to David Lynch's work as marginal cinema, our analysis is based on the semio-pragmatic theory. Through a selection of three films by this great director, the goal of this article is to apprehend the interpretative leeway given to the spectator, thanks to the whole set of tools provided by this heuristic model.

\section{INDEX}

Keywords : margin, interpretation, narration, semio-pragmatics, David Lynch

Mots-clés : marge, interprétation, narration, sémio-pragmatique, David Lynch

\section{AUTEUR}

\section{JEAN-MICHEL DENIZART}

Aix-Marseille Université

Jean-michel.denizart@univ-amu.fr

Travail de recherche bénéficiant du soutien financier du Conseil régional Provence-Alpes-Côte d'Azur. 